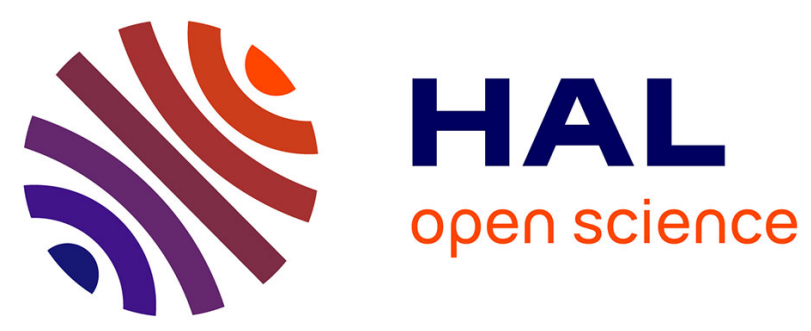

\title{
High-frequency simultaneous measurements of velocity and concentration within turbulent flows in wind-tunnel experiments
}

\author{
M. Marro, H. Gamel, P. Méjean, H. Correia, L. Soulhac, P. Salizzoni
}

\section{> To cite this version:}

M. Marro, H. Gamel, P. Méjean, H. Correia, L. Soulhac, et al.. High-frequency simultaneous measurements of velocity and concentration within turbulent flows in wind-tunnel experiments. Experiments in Fluids, 2020, 61 (12), 10.1007/s00348-020-03074-7 . hal-02991472

HAL Id: hal-02991472

https://hal.science/hal-02991472

Submitted on 6 Nov 2020

HAL is a multi-disciplinary open access archive for the deposit and dissemination of scientific research documents, whether they are published or not. The documents may come from teaching and research institutions in France or abroad, or from public or private research centers.
L'archive ouverte pluridisciplinaire HAL, est destinée au dépôt et à la diffusion de documents scientifiques de niveau recherche, publiés ou non, émanant des établissements d'enseignement et de recherche français ou étrangers, des laboratoires publics ou privés. 


\title{
High-frequency simultaneous measurements of velocity and concentration within turbulent flows in wind-tunnel experiments
}

\author{
M. Marro ${ }^{1}$ - H. Gamel ${ }^{1}$. P. Méjean ${ }^{1}$ - H. Correia ${ }^{1}$. L. Soulhac ${ }^{1}$. \\ P. Salizzoni ${ }^{1}$
}

\begin{abstract}
We analyse the metrological aspect related to systems devoted to the simultaneous measurements of (one point) velocity and scalar concentration statistics. We consider systems based on the coupling of an anemometer (Laser Doppler or Hot Wire) with a Flame Ionization Detector. In particular, we focus on the estimate of the cross-correlation velocity-concentration. Key aspects in the reliability of such measurements are related to the identification of an optimal distance between the two probes, the time lag with which the velocity and concentration signals are acquired, and, eventually, the influence of seeding particles (when using a Laser Doppler Anemometer) on the concentration measurements. We investigate these aspects by performing wind-tunnel experiments on the scalar dispersion down wind a ground-level source in a turbulent boundary layer. This analysis allowed us to identify a time delay and a convenient distance between the signals provided by the anemometer and the concentration detector. Results also show that the seeding used for the Laser Doppler Anemometer measurements modifies the cut-off frequency in the concentration spectra and induces a slightly larger uncertainty on the statistics of scalar field. The results show the reliability of both systems in estimating (horizontal and vertical) turbulent mass fluxes.
\end{abstract}

M. Marro

E-mail: marro.massimo@ec-lyon.fr

P. Salizzoni

E-mail: pietro.salizzoni@ec-lyon.fr

${ }^{1}$ Laboratoire de Mécanique des Fluides et d'Acoustique, University of Lyon, CNRS UMR 5509 Ecole Centrale de Lyon, INSA Lyon, Université Claude Bernard, 36, avenue Guy de Collongue, 69134 Ecully, France
Keywords Flame Ionization Detection · Hot-Wire Anemometry · Laser Doppler Anemometry · Turbulent Mass Fluxes · Wind-Tunnel Experiments

\section{Introduction}

The experimental estimate of joint velocity-concentration statistics is a key issue for the study of the dispersion and mixing of scalar within a turbulent flow. This requires simultaneous measurements of the flow velocity and the scalar field, which generally imply to couple two separate acquisition systems. The use of Particle Image Velocimetry (PIV) combined to other optical techniques for the concentration measurements is well suited in water flume experiments (e.g., Liao and Cowen, 2002). Its use in wind-tunnel tests however (Vinçont et al, 2000), still presents main shortcomings, mainly concerning the control of the seeding particles emitted by the source (which explains the relatively low number of study published so far adopting these techniques).

The most reliable and robust techniques rely so far in the adoption of different metrological approaches for the measurements of combined one-point statistics. In the study of scalar dispersion within a turbulent boundary layer, several authors have combined a X-probe Hot-Wire Anemometer (HWA) (providing statistics of two velocity components) with another technique in order to measure the concentration of a scalar (that could be either a chemical species or the temperature). In their heavily cited work, Fackrell and Robins (1982) coupled a HWA and Flame Ionization Detection (FID) to characterise the dispersion downwind a localised release of propane. In a similar experimental configuration, a same HWA-FID coupling has been subsequently used by Koeltzsch (1999) and by Iacobello et al (2019). 
A slightly different approach was instead adopted by Metzger and Klewicki (2003) and by Talluru et al (2018) who used a Photoionisation Detector for the concentration measurements (a system with a slightly lower resolution in frequency, compared to a FID).

To investigate dispersion downwind an elevated linear source, instead of releasing a chemical species, it may be convenient to use the temperature as passive scalar, since the line source can be easily designed as a suspended electric resistance providing a constant heat flux (heated by Joule effect and cooled by turbulent convection). With this experimental setup Raupach and Legg (1983) and Stapountzis et al (1986) used a 3-wire probe to measure the velocity-temperature correlations: 2 hot-wires and a cold wire for the measurements of two velocity components and the temperature, respectively.

The approaches mentioned above have the same main shortcoming: the HWA is not suited for measurements within recirculating flows, in high turbulence intensity flow regions (i.e. shear layers) and its intrusiveness can modify the motion field. For these reasons these approaches can not be used to investigate scalar transport within and above complex geometries, typically represented by idealised urban or vegetation canopies.

These limitations can be overcome by replacing the HWA with a Laser Doppler Anemometry (LDA) and coupling the latter with a FID. This approach was actually used to investigate the scalar dispersion within urban-like geometries (Contini et al, 2006; Carpentieri and Robins, 2010; Carpentieri et al, 2012; Kukačka et al, 2012; Nosek et al, 2016; Marucci and Carpentieri, 2020). Even the adoption of a LDA-FID system however has to face some metrological problems, namely to:

1. evaluate the influence of the aerosol particles used to seed the flow on the FID measurements;

2. compute reliable estimates of cross-correlations from signals (velocity and concentration) shifted in time and with different sampling frequencies;

3. determine an optimal distance between the FID probe and the measurement volume of the LDA;

4. evaluate the time lag with which the velocity and concentration signals are actually acquired by the two instruments.

It is worth noting that the latter point is also of concern for the use of a HWA-FID system.

Despite the non-negligible number of study adopting these approaches, an analysis of these metrological aspects has not been presented so far in the literature. To fill this gap we performed a parametric study, in order to test and estimate the accuracy of scalar massflux measurements, as obtained by both (LDA-FID and HWA-FID) systems. After presenting the setup of the experiments and giving details on the adopted measurement techniques (Sect. 2), we provide the main features of the velocity field within which the dispersion took place (Sect. 3). We then focus on the LDA-FID system (Sect. 4), and specifically address the four points raised before. Finally, we compare the results with those obtained through a HWA-FID system and evaluate the accuracy of both systems in determining the turbulent mass fluxes (Sect. 5).

\section{Experimental setup and measurement techniques}

The experiments were performed in the atmospheric wind tunnel of the Laboratoire de Mécanique des Fluides et d'Acoustique at the Ecole Centrale de Lyon in France. This is a recirculating wind tunnel with a working section measuring $9 \mathrm{~m}$ long, $0.7 \mathrm{~m}$ wide, and $1 \mathrm{~m}$ high. A neutrally-stratified boundary layer was generated by combining the effect of a row of spires placed at the beginning of the test section, and roughness elements on the floor (Fig. 1a). The spires were of the Irwin (1981) type with a height of $0.5 \mathrm{~m}$. The entire working section floor was overlaid with square section sticks of length $l=0.014 \mathrm{~m}$ as roughness elements, spaced by a distance $3 l$, and placed normal to the flow direction. This experimental setup allowed a boundary layer of a depth $\delta=0.55 \mathrm{~m}$ to be reproduced. Imposing a free-stream velocity $u_{\infty}=6.33 \mathrm{~m} \mathrm{~s}^{-1}$, the Reynolds number $R e=\delta u_{\infty} / \nu \approx 2.3 \times 10^{5}$ (being $\nu \approx 1.52 \times 10^{-5}$ $\mathrm{m}^{2} \mathrm{~s}^{-2}$ the air kinematic viscosity) was sufficiently high to ensure the adequate simulation of a fully turbulent flow (Jimènez, 2004).

In the passive scalar experiments, ethane $\left(\mathrm{C}_{2} \mathrm{H}_{6}\right)$ was continuously released from a ground-level line source and used as a tracer, since its density is similar to that of air. The source consisted of a metallic tube pierced with needles producing well mixed ethane-air release within a cavity of $0.01 \mathrm{~m} \times 0.01 \mathrm{~m}$ size, as shown in Fig. 1b. The flow control system at the source was composed of two lines, ethane and air, each of them equipped with a mass flow controller. The two lines then converged through a valve and the ethane-air mixture was directed to the source. The volume flow $Q_{t o t}$ was equal to $1.67 \times 10^{-4} \mathrm{~m}^{3} \mathrm{~s}^{-1}$, resulting in an injection velocity $u_{s} \approx 0.024 \mathrm{~m} \mathrm{~s}^{-1}$ (see Fig. 1b). The ethane mass rate was kept constant by the mass flow

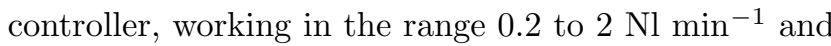
used within 10 and $100 \%$ of its nominal range. The error on the ethane-air flow rate was estimated by systematic comparison with measurements provided by a volumetric counter. The maximal difference between mea- 
surements of the two instruments did not exceed $\pm 3 \%$ (Nironi et al, 2015).
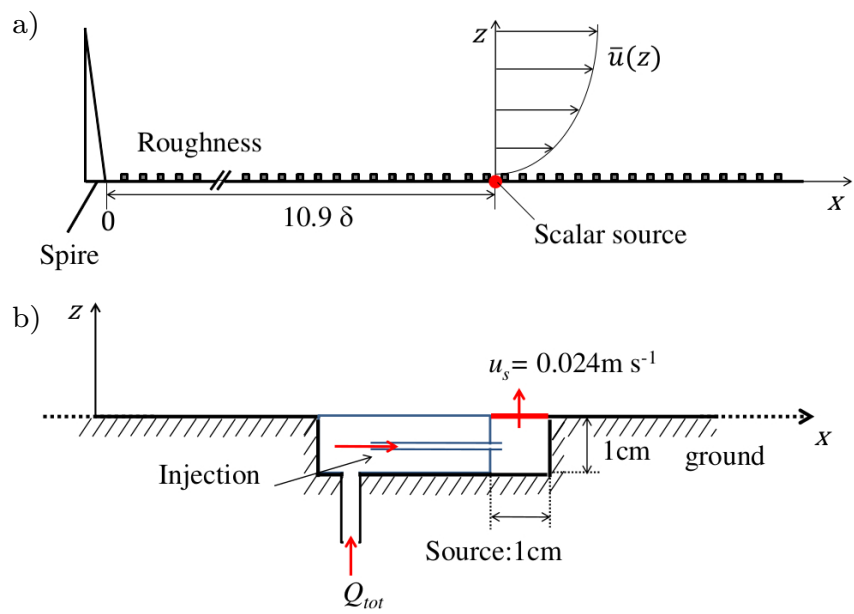

Fig. 1 Wind-tunnel setup: a) sketch of the experimental boundary layer, b) detail of the linear source

\subsection{LDA}

The Laser Doppler Anemometer was equipped with a $5 \mathrm{~W}$ power laser, producing two blue and two green beams in perpendicular planes with wavelength $\lambda_{\text {blue }}=$ $488 \mathrm{~nm}$ and $\lambda_{\text {green }}=514.5 \mathrm{~nm}$, respectively. The beams have a diameter of $0.1 \mathrm{~mm}$ and the front lens presents a focal length of $400 \mathrm{~mm}$. The sample volume was estimated as a function of the focal length, the initial beam thickness, and the laser wavelength (e.g., Jensen, 2004). The measurement volume of the blue beam is defined by $\Delta x=0.11 \mathrm{~mm}, \Delta y=0.11 \mathrm{~mm}$, and $\Delta z=2.3 \mathrm{~mm}$, whereas for the green beam $\Delta x=0.12 \mathrm{~mm}, \Delta y=0.12$ $\mathrm{mm}$, and $\Delta z=2.4 \mathrm{~mm}$. Note that a significant source of uncertainty is the position of the measuring volume that we estimated as about $\pm 1.0 \mathrm{~mm}$ for the three directions. The flow was seeded with olive oil (Boskou et al, 2006) injected by means of an atomizer generating polydisperse drops of typical diameter of about $1 \mu \mathrm{m}$. The seeding occurred before the entrance of the wind tunnel, upwind the spires location (about 8 meters before the measuring section).

In order to provide insight on the LDA sampling frequency compared to typical time scales of the flow, we show in Fig. 2 the vertical profile of the data density. This latter parameter represents the ratio of the mean time that takes the instantaneous velocity to change by one standard deviation to the mean time between samples (Adrian and Yao, 1987) and it is defined as

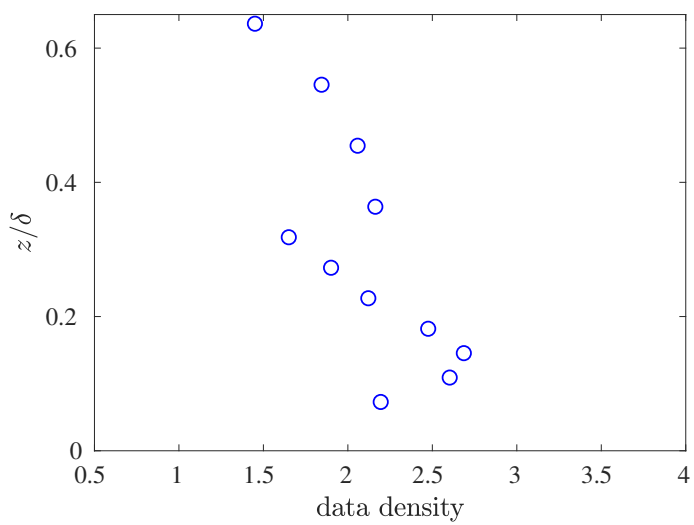

Fig. 2 Vertical profile of the data density

the samples collected on average during one Taylor microscale $T_{\lambda}$ of the velocity signal (Adrian, 1983, p. 246). We computed the data density as the product $\dot{N} T_{\lambda}$, where $\dot{N}$ is the mean data rate. The Taylor microscale was here estimated as (e.g., Adrian and Yao, 1987):

$T_{\lambda}=\sigma_{u} / \sqrt{\left\langle\left(\frac{d u}{d t}\right)^{2}\right\rangle}$,

where the brackets denote the ensemble average of the enclosed expression, and $\sigma_{u}$ (whose vertical profile is reported in Fig. 3b) is the standard deviation of the longitudinal (instantaneous) velocity $u$.

As shown in Fig. 2, the values of the data density across the vertical extent of the boundary layer flow are in the range 1.4-2.7, which represents an "intermediate" data density (Edwards, 1987).

\subsection{HWA}

We used a hot-wire constant temperature anemometer equipped with a $\mathrm{X}$-wire probe with a velocity-vector acceptance angle of $\pm 45^{\circ}$, allowing for the simultaneous measurements of two velocity components. Calibration was carried out in the wind tunnel using a Pitot tube to measure a reference velocity. The probe was not calibrated in yaw. In order to decompose the calibration velocities from the $\mathrm{X}$-probe into the longitudinal and transversal velocity components (Jorgensen, 2002), we adopted a yaw correction with constant coefficients $k_{1}^{2}=k_{2}^{2}=0.0225$. The temperature was constantly monitored by means of a type $\mathrm{K}$ thermocouple placed close to the probe. In case of temperature variation larger than $2^{\circ} \mathrm{C}$ during an experiment, a new calibration was performed. 


\subsection{FID}

Concentration measurements were performed with a Flame Ionization Detector (Fackrell, 1980) using a sampling tube $0.3 \mathrm{~m}$ long, permitting a frequency response of the instrument to about $800 \mathrm{~Hz}$. Vertical and transverse (not shown here) profiles of concentration statistics were recorded at various distances downwind, from $x=0.33 \delta(x=182 \mathrm{~mm})$ up to $x=2.18 \delta(x=1200$ $\mathrm{mm})$. The calibration was carried out using ethane-air mixtures with concentrations equal to $0,500,1000$, and $5000 \mathrm{ppm}$. As a general rule, calibration was performed twice a day. When the flame temperature showed variations of more than $2^{\circ} \mathrm{C}$ from the value recorded at the beginning of the experiment, calibration was repeated. The relation between ethane concentration and voltage response was linear, with a slope (representing the sensitivity of the instrument) whose variations could reach $\pm 3 \%$, depending on the ambient conditions.

Recirculation of air in the wind tunnel implies background concentration increasing with time. To take into account the contribution of this drift, the background concentrations were recorded before and after each concentration time series. The background concentration, which was assumed to evolve linearly with time from its initial to its final value, was then subtracted from the signals.

\section{Velocity field}

The statistics of the velocity field, measured both with a LDA and HWA system, are shown in Fig. 3. The HWA measurements were performed over the whole boundary-layer depth, whereas the LDA measurements were instead limited to about $z \approx 0.6 \delta$. As mentioned in Sect. 2.1, all the velocity statistics computed with LDA data were weighted on the transit time (Edwards, 1987).

Furthermore, we diagnosed the transit times $\tau_{n}$ since commercial LDA-processors could provide values that are not reliable (George, 1988). To that purpose we analysed the scatter plots of the velocity versus $\tau_{n}$ (not shown here) reporting the characteristic shape (generally observed for positive convection velocity and moderate turbulence intensity, and usually referred to as "banana" shape) that highlights a correlation between short residences times and high velocities (and viceversa) (Velte et al, 2014; Yaacob et al, 2020).

The two techniques - HWA and LDA - provided very similar vertical profiles for the the mean $\bar{u}$ (Fig. 3a) and the standard deviation $\sigma_{u}$ (Fig. 3b) of the longitudinal velocity component. Some differences can instead be observed in $\sigma_{w}$ (Fig. 3b), namely close to the ground, where the HWA sliglty underestimates $\sigma_{w}$ values compared to the LDA. Measurements of $\sigma_{v}$ were performed with HWA only. The main differences between the two techniques concern the values of the Reynolds stresses $\overline{u^{\prime} w^{\prime}}$. Notably, close to ground level, i.e. for $z / \delta<0.3$, the Reynolds stresses measured by LDA are significantly larger than those obtained by HWA (see Fig 3c). This is due to well-documented shortcomings of the HWA in flows with high turbulence intensity $\sigma_{u} / \bar{u}$ (Tutu and Chevray, 1975; Kawall et al, 1983; Perry et al, 1987). Tutu and Chevray (1975) showed that the error in the estimate of $\overline{u^{\prime} w^{\prime}}$ might reach $28 \%$ for $\sigma_{u} / \bar{u}>$ $35 \%$. According to Kawall et al (1983), this effect is due to the influence of the fluctuations of the transverse velocity $v$, that becomes the main source of error as $\sigma_{u} / \bar{u}>15 \%$. Perry et al (1987) showed that an increasing of the included angle of the $\mathrm{X}$-wires allowed the error to be partially reduced, but with a significant deterioration in the estimate of the normal fluctuating component $w$ as a secondary effect. Our HWA measurements are consistent with the conclusions of Kawall et al (1983) who showed that the error in the estimates of $\overline{u^{\prime} w^{\prime}}$ varied between $5 \%$ and $40 \%$ for turbulent intensities in the range $15 \%<\sigma_{u} / \bar{u}<40 \%$ (see Fig. $3 \mathrm{~d}$ ). Based on these velocity measurements, we could estimate the friction velocity $u_{*}=\sqrt{-\overline{u^{\prime} w^{\prime}}}=0.054 u_{\infty}$ from LDA data (Fig. 3c), and the roughness length $z_{0}=0.0024 \delta$ by fitting a logarithmic law to the mean longitudinal velocity profile (Tennekes, 1982). In both cases we referred to data collected in the lower part of the boundary layer, i.e. $z / \delta \leq 0.25$.

The other flow variable that will be useful in our following analysis is the vertical Eulerian integral length scale $L_{w w}$, defined as:

$L_{w w}(z)=\int_{0}^{\infty} \frac{w^{\prime}(z) w^{\prime}(z+r)}{\sigma_{w}^{2}} d r$,

with $r$ a generic distance in the vertical direction. In principle, estimating $L_{w w}(z)$ would require simultaneous two-point velocity measurements to be estimated (for instance, using a PIV system). Alternatively an estimate of $L_{w w}=L_{w w, s p}$ can be obtained by evaluating the wave number at which maxima of vertical velocity spectra occur (Pope, 2013, p. 226). In the upper part of the boundary layer the peaks of the spectra flatten out and, therefore, the evaluation of $L_{w w}$ is affected by a large uncertainty (the error bars in Fig. 3e corresponding to an uncertainty of $5 \%$ in the evaluation of the maximum of the vertical velocity spectrum).

In Fig. 3e we also compare our estimates of $L_{w w}$ with the values provided by Robins (1979) for two neutral boundary layers, referred to as BL1 and BL2, characterised by a different wall roughness: $z_{0} / \delta=0.0004$ 

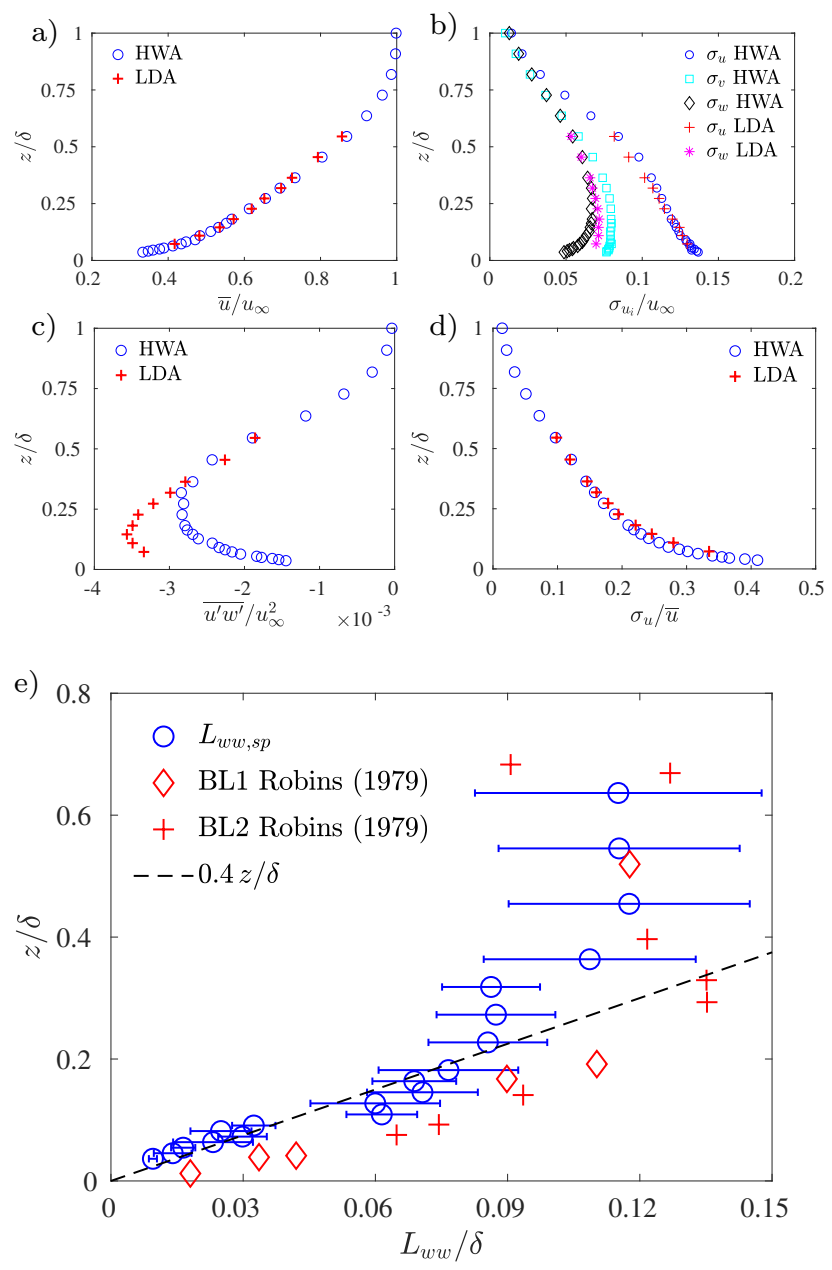

Fig. 3 Vertical profiles of the a) mean longitudinal velocity $\bar{u}, \mathrm{~b})$ standard deviation of the three velocity components, c) vertical Reynolds stress $\overline{u^{\prime} w^{\prime}} / u_{\infty}^{2}$, d) the local turbulence intensity $\sigma_{u} / \bar{u}$, e) Eulerian integral length scale $L_{w w}$ and associated error bars (see text).

for BL1 and $z_{0} / \delta=0.022$ for BL2. Note that, according to the statements of the similarity theory, the vertical evolution of $L_{w w}$ is not sensitive to the wall roughness and depends on the distance on the wall only. Notably, in the lower part of the boundary layer the vertical evolution of $L_{w w}$ is well approximated by the relation $L_{w w}=0.4 z$, as predicted by the Prandtl mixing length theory (Schlichting, 1979).

\section{LDA-FID coupling}

We developed a system to provide simultaneous measurements of the velocity and concentration fields by means of a coupling between LDA and FID (see sketch in Fig. 4). In order to investigate the reliability of a such experimental setup, we analyse the four aspects that we have already pointed out in the introduction, notably: i)

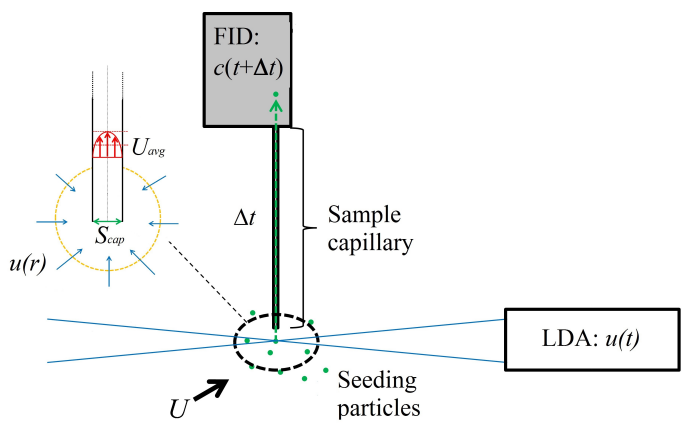

Fig. 4 LDA-FID system

the influence of the aerosols on the FID measurements (Sect. 4.1); ii) the choice of a suitable method to shift and resample velocity and concentration signals to compute cross-correlations (Sect. 4.2); iii) the evaluation of an optimal distance between the FID probe and LDA measurement volume and iv) of the time lag between the signals acquired by the two instruments (Sect. 4.3).

4.1 Influence of LDA seeding particles on FID concentration measurements

The olive oil drops (Boskou et al, 2006) used to seed the flow potentially perturb the concentration measurements performed by the FID system, which is sensitive to the presence of organic carbon in air. For this reason we analysed the concentration signals and the spectra for a flow with and without oil drops (Fig. 5). In particular we observe that, in this example, the FID provides concentration values with a mean value equal to about $1 \mathrm{ppm}$ and fluctuations of $\pm 1.0-2.0 \mathrm{ppm}$ (standard deviation equal to $0.34 \mathrm{ppm}$ ) for a flow without oil drops. On the contrary, the presence of seeding particles induces some high concentration peaks of about 20-30 ppm with a standard deviation of about $1.2 \mathrm{ppm}$. This is consistent with the findings of Kukačka et al (2012) who investigated the effects of seeding the flow field (glycerine droplets) on the signals collected by a FID. They argued that the presence of drops induced very rare spikes in the time series that had no relevant effect on the concentration statistics. The influence of the oil drops is also evident on the concentration spectra: the cut-off frequency is between 400 and $500 \mathrm{~Hz}$ without oil, whereas the seeding moves it between 300 and $400 \mathrm{~Hz}$, showing that the signal bandwidth is mainly distributed on the higher frequencies.

The analysis of the concentration statistics allows the impact of seeding on the scalar field to be investigated in more detail. Figure 6 reports the vertical 


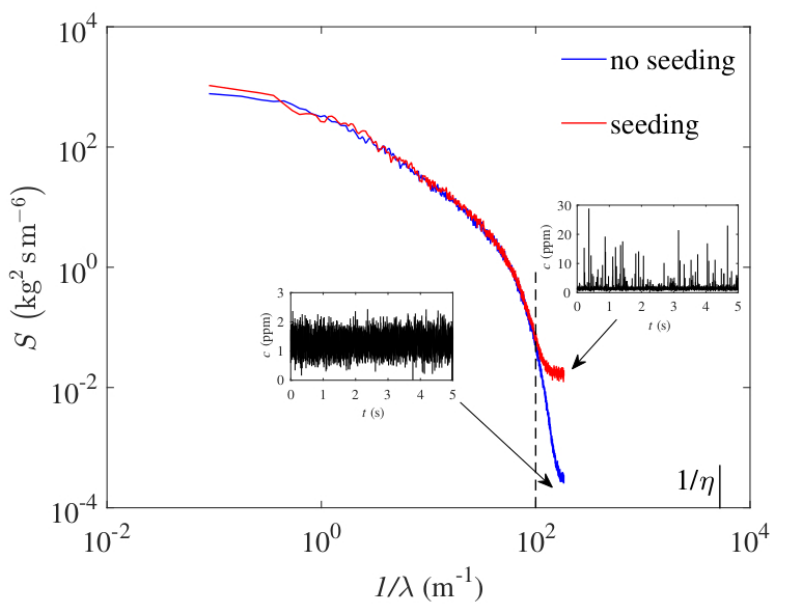

Fig. 5 Influence of the olive oil drops on the concentration signals measured by FID at $z=0.075 \delta$; $\eta$ represents an estimate of the Kolmogorov length scale

profiles of the first four moments of the concentration centered around the mean $\bar{c}$ for a sampling time equal to $300 \mathrm{~s}$ varying the distance from the source location. The concentration statistics are normalized as:

$m_{i}^{*}=\left[\frac{1}{N_{s}} \sum_{k=1}^{N_{s}}\left(c_{k}-\bar{c}\right)^{i}\right]^{1 / i} \frac{u_{\infty} \delta}{Q_{t o t} / L} \quad i=2,3,4$,

where $N_{s}$ is the number of the instantaneous concentration values $c_{k}$ collected in a measurement point and $Q_{\text {tot }} / L$ is the linear mass flow of the passive scalar. In the following notation $m_{2}^{*}$ is referred to as $\sigma_{c}^{*}=m_{2}^{*}$.

We observe that the high concentration peaks due to the oil drop seeding slightly increase on the higherorder concentration statistics, namely on the estimates of $\sigma_{c}^{*}, m_{3}^{*}$, and $m_{4}^{*}$ (Fig. 6). Nevertheless, this influence is generally low and the differences are quite slight for all the statistics.

To quantify the impact of the presence of oil droplets on the accuracy and repeatability of the concentration measurements we estimate the uncertainty on the scalar field statistics computed over two series of 30 measurements, with and without the flow seeding. For each series we have then computed the ensemble average $E_{i}$ and the normalized standard deviation $S_{i}$ (in \%) of the first four moments of the concentration that represents an estimate of the uncertainty of measurement (JCGM, 2008). In Table 1 we report the data acquired at three different measurement positions, in the near (P1), intermediate (P2), and far field (P3). For the measurements without the presence of seeding particles, the values of $S_{i}$ are in general in the range $1 \% \lesssim S_{i} \lesssim 3 \%$ for all four concentration moments, which is agreement with previous estimates (Nironi et al, 2015). Uncertainty of measurement in presence of oil seeding particles is however larger and falls in the range $2 \% \lesssim S_{i} \lesssim 5 \%$. This analysis showed that the oil drops induced a slightly larger dispersion of the measurements, but their influence on the concentration statistics remained low.

\subsection{Cross-correlation estimate}

In estimating the turbulent mass fluxes $\overline{u^{\prime} c^{\prime}}$ and $\overline{w^{\prime} c^{\prime}}$ we set the acquisition system (LDA-FID) in order to be governed by the LDA, i.e. the velocity and the concentration signals were simultaneously collected when the seed particles crossed the LDA sample volume. As a consequence of that, the sampling frequency of the concentration is also irregular. Since there is a time delay between the measurements obtained by LDA and FID (discussed in Sect. 4.3), we have to adopt a suitable method to compute the cross-correlations between velocity and concentration signals. We tested two different methods, the slot correlation technique (Mayo, 1975; Müller et al, 1998; Nobach, 2016) and the sample-andhold reconstruction and resampling $(\mathrm{S}+\mathrm{H})$. The former is a well-established technique allowing reliable estimates of the cross-correlation to be obtained for mixed independent and dependent sampling (Nobach, 2016). The latter is a simpler method that consists of the following items:

- shifting the FID signal of a suitable time delay $\Delta t_{\text {lag }}$;

- resampling the concentration values on the temporal pattern of LDA data, i.e. on the velocity signals, (Fig. 7a);

- computing the cross-correlation with transit-time weighting (similarly to what presented in Nobach et al (1998)).

Note that the $\mathrm{S}+\mathrm{H}$ method is straightforward to implement and it has the advantage to require a very low computational cost. Although this method was applied to the concentration signals in several works (e.g. Contini et al, 2006; Kukačka et al, 2012; Marucci and Carpentieri, 2020) to estimate the cross-correlations, a real discussion about the reliability of the results was not usually tackled. Figure $7 \mathrm{~b}$ shows that the turbulent mass fluxes computed with the two methods are generally in good agreement, even though differences of about $6 \%$ occur for some points.

4.3 Estimate of the optimal distance and time lag between LDA and FID

Ideally, the FID probe should be placed as closest as possible to the measurement volume of the LDA. How- 
a)

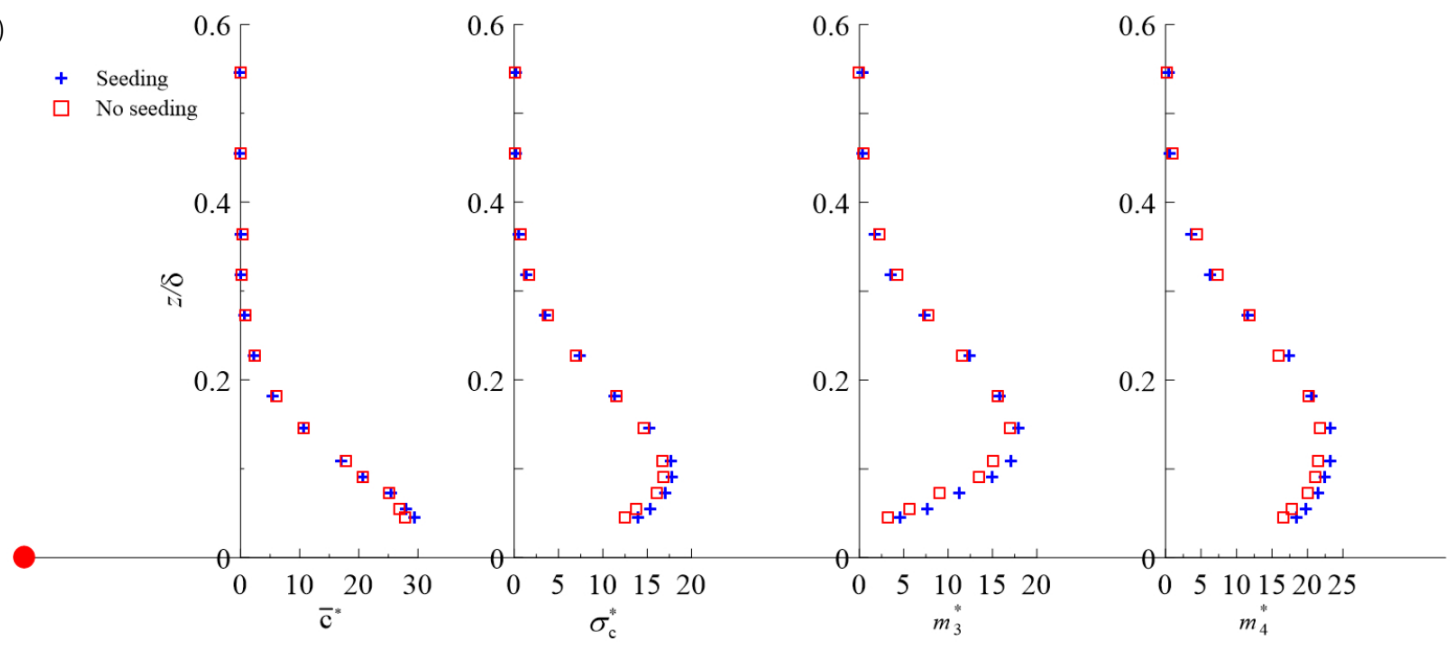

b)

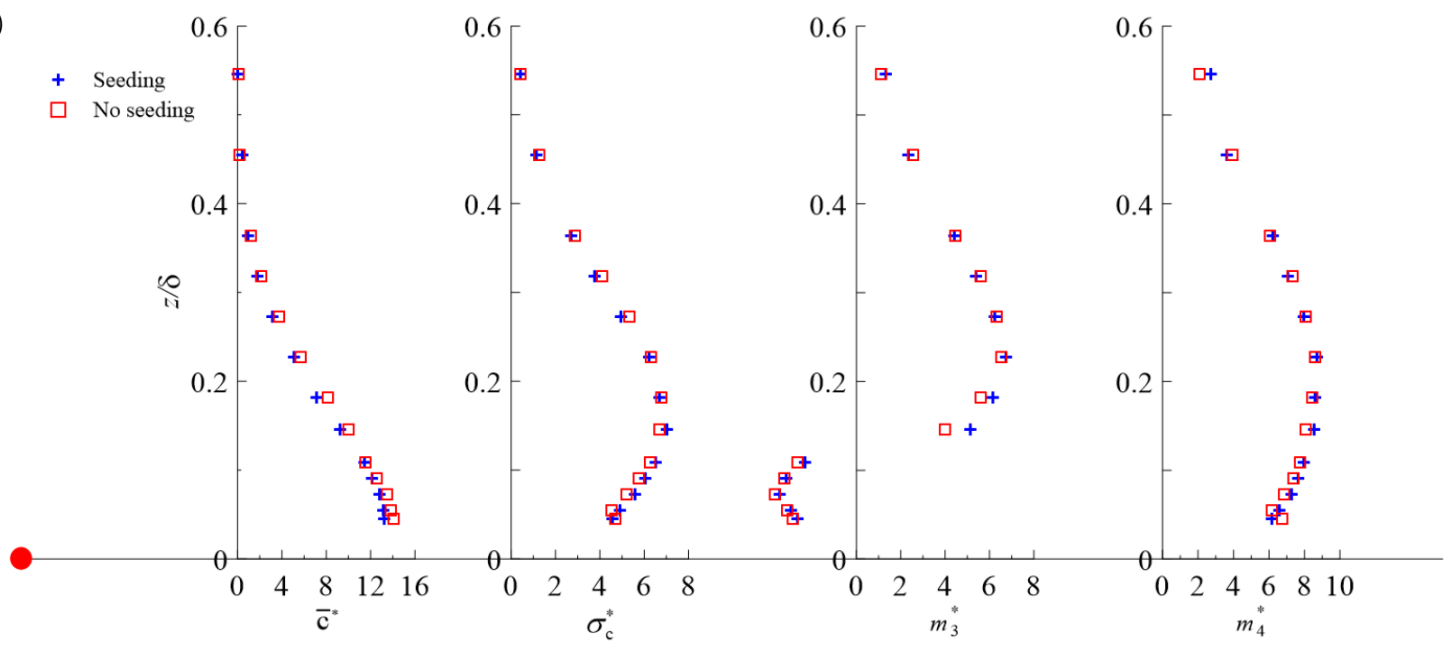

Fig. 6 Influence of seeding on the vertical profiles of the concentration statistics at different distances from the source location: a) $x=0.75 \delta$, b) $x=1.75 \delta$
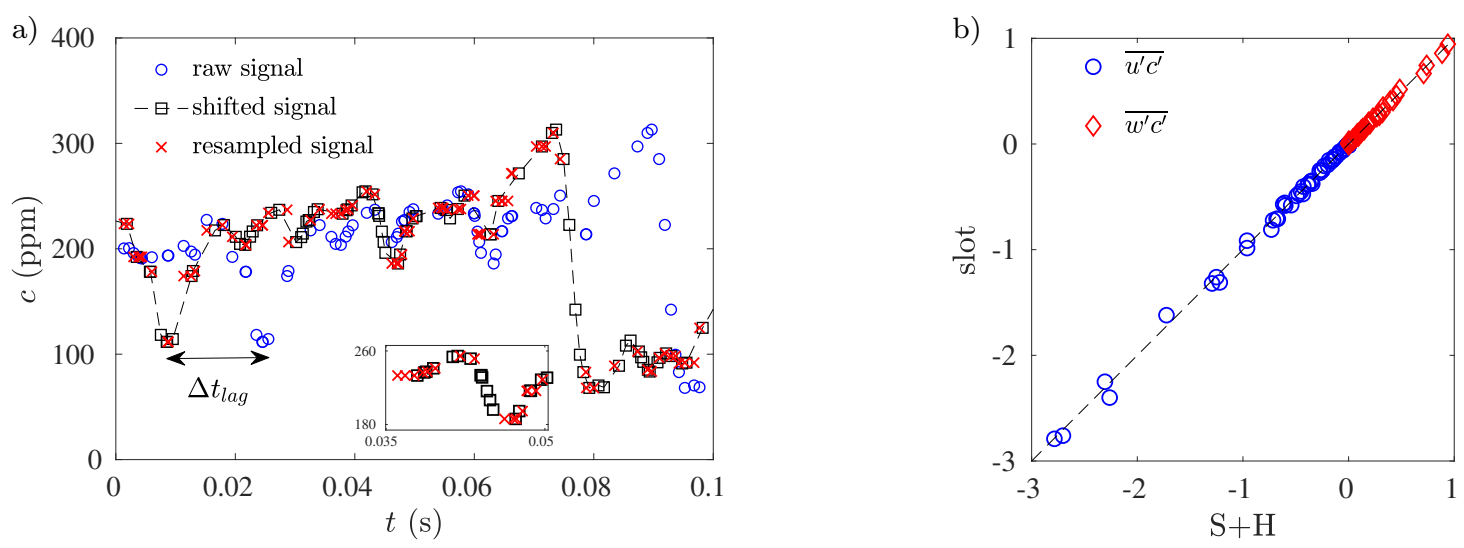

Fig. 7 Sample-and-hold reconstruction and resampling method $(\mathrm{S}+\mathrm{H})$ : a) raw, shifted $\left(\Delta t_{\text {lag }}\right.$ is the time delay), and resampled FID signals, b) scatter plot of the cross-correlations $\overline{u^{\prime} c^{\prime}}$ and $\overline{w^{\prime} c^{\prime}}$ computed by means of the slot correlation technique and $\mathrm{S}+\mathrm{H}$ method 


\begin{tabular}{|c|c|c|c|c|c|c|c|c|c|}
\hline \multirow{2}{*}{ Point } & Variable & \multicolumn{2}{|c|}{$\bar{c}$} & \multicolumn{2}{c|}{$\sqrt{\bar{c}^{\prime 2}}$} & \multicolumn{2}{c|}{$\sqrt[3]{\bar{c}^{\prime 3}}$} & \multicolumn{2}{c|}{$\sqrt[4]{\overline{c^{\prime 4}}}$} \\
\cline { 2 - 10 } & & no seeding & seeding & no seeding & seeding & no seeding & seeding & no seeding & seeding \\
\hline \multirow{2}{*}{ P1 } & $E_{i}$ & 3.97 & 3.93 & 3.04 & 3.03 & 2.60 & 2.60 & 4.11 & 4.10 \\
\cline { 2 - 10 } & $S_{i}(\%)$ & 1.4 & 3.9 & 1.1 & 3.5 & 1.9 & 1.9 & 1.6 & 3.4 \\
\hline P2 & $E_{i}$ & 1.75 & 1.75 & 0.96 & 0.96 & 0.30 & 0.35 & 1.19 & 1.21 \\
\cline { 2 - 10 } & $S_{i}(\%)$ & 1.5 & 3.1 & 1.1 & 2.4 & 3.3 & 2.5 & 1.2 & 2.5 \\
\hline P3 & $E_{i}$ & 0.71 & 0.71 & 0.59 & 0.59 & 0.49 & 0.50 & 0.73 & 0.74 \\
\cline { 2 - 10 } & $S_{i}(\%)$ & 2.2 & 4.5 & 1.1 & 3.5 & 1.2 & 3.0 & 1.0 & 3.3 \\
\hline
\end{tabular}

Table 1 Repeatability of concentration measurements and influence of seeding on moments of the concentration: ensemble average $E_{i}$ and normalized standard deviation $S_{i}$ (in \%)

ever, the FID is an intrusive method performing measurements by aspiring the fluid to a flame chamber, which implies local modifications of the flow field. We need therefore to evaluate an optimal distance $\Delta x_{i}$ between the LDA and FID, that has to fulfill two constraints:

- be sufficiently large to avoid flow perturbation induced by the FID system on the LDA measurement volume;

- be sufficiently small so that the measurements of the concentration and the velocity can be considered as representative of a same fluid element.

Moreover, $\Delta x_{i}$ introduces a time lag $\Delta t_{\text {lag }}$ between the velocity and concentration acquisitions (Fig. 4) that alters the estimates of $\overline{u^{\prime} c^{\prime}}$ and $\overline{w^{\prime} c^{\prime}}$. This effect is further amplified by the sample travel time into the capillary until the flame chamber. To minimize this, we applied the techniques presented in Sect. 4.2.

First indications about approximate values of $\Delta x_{i}$ and $\Delta t_{\text {lag }}$, can be obtained based on some characteristics of the FID and using some simple relations. To compute the travel time $\Delta t_{l a g}$ we need some characteristics of the FID, as the capillary length $L_{c}=0.3$ $\mathrm{m}$, its radius $r_{c}=0.005 \mathrm{in} \approx 1.27 \times 10^{-4} \mathrm{~m}$, and the imposed pressure drop $\Delta p_{c}=33330.6 \mathrm{~Pa}$. The mean travel velocity $\bar{u}_{c}$ can be estimated from the pressure drop along a smooth pipe:

$\Delta p=\frac{L_{c}}{2 r_{c}} \frac{\rho_{a i r} \bar{u}_{c}^{2}}{2} \lambda_{f}$

where $\rho_{\text {air }} \approx 1.18 \mathrm{~kg} \mathrm{~m}^{-3}$ is the air density at ambient temperature and $\lambda_{f}$ is the head-loss coefficient. In laminar flow the Poiseuille law states $\lambda_{f}=64 / R e_{c}$, where $R e_{c}=\bar{u}_{c} 2 r_{c} / \nu$ is the Reynolds number. With these assumptions we obtain $\bar{u}_{c}=12.5 \mathrm{~m} \mathrm{~s}^{-1}$ (from Eq. 4) and, therefore, $\Delta t_{\text {lag }} \approx L_{c} / \bar{u}_{c}=24 \mathrm{~ms}$, and $R e=208$ (we therefore verify the hypothesis of laminar flow). Here, we approximated the velocity profile $\bar{u}_{c}$ as uniform. If we consider that in a circular pipe the velocity profile presents a parabolic shape and, therefore, the maximum velocity is twice the mean velocity, the travel is reasonably included in the range between 12 and $24 \mathrm{~ms}$.
In order to estimate the magnitude of $\Delta x_{i}$, we consider that the volume flow rate in the capillary is equal to that crossing the spherical surface of radius $r_{s}$ enveloping the FID inlet (see Fig. 4):

$\bar{u}_{c} \pi r_{c}^{2}=4 \pi r_{s}^{2} \bar{u}_{s}\left(r_{s}\right)$

where $\bar{u}_{s}\left(r_{s}\right)$ is the mean radial velocity. Based on Eq. 5 we compute the distance at which $\bar{u}_{s}\left(r_{s}\right)$ is two order of magnitude lower than the mean flow velocity. In our experiments we satisfy this condition - i.e. $\bar{u}_{s}<0.125$ $\mathrm{m} \mathrm{s}^{-1}$ - for $r_{s}>0.63 \mathrm{~mm}$, which, therefore, can be considered as the minimal $\Delta x_{i}$ to be imposed.

Of course, the values previously reported have to be considered just as reference estimates of $\Delta t_{\text {lag }}$ and $\Delta x_{i}$. To properly identify their optimal values we performed a sensitivity analysis imposing a variable distance between the two measurements points in the directions $x$, $y$, and $z$, separately.

Similarly, Metzger and Klewicki (2003) investigated the influence of suction of a photoionisation detector focusing on the effects on the velocity statistics measured with a HWA. They found that an aspiration speed of about $0.2 u_{\infty}$ could affect the estimates of velocity statistics, especially the Reynolds stress, in the lower part of the boundary layer.

We performed several measurements in the point $(x \approx 1.1 \delta, y=0, z \approx 0.075 \delta)$ for increasing distances $\Delta x_{i}$ (being $\Delta x_{1}=\Delta x, \Delta x_{2}=\Delta y$, and $\Delta x_{3}=\Delta z$ ) and then we applied the slot correlation technique (see Sect. 4.2) to compute the correlation coefficients $R \overline{u_{u^{\prime} c^{\prime}}}$ and $R \overline{\overline{w^{\prime} c^{\prime}}}$ as a function of $\Delta t_{l a g}$ (Fig. 8):

$R \overline{u_{i}^{\prime} c^{\prime}}=\frac{\overline{u_{i}^{\prime}(t) c^{\prime}(t)}}{\sigma_{u_{i}} \sigma_{c}}, \quad i=1,3$,

where $\sigma_{u_{i}}$ and $\sigma_{c}$ are, respectively, the standard deviation of the velocity and of the concentration computed in a measurement point, and $u_{1}^{\prime}=u^{\prime}, u_{3}^{\prime}=w$. The distance $\Delta x_{i}$ was normalized with the value of Eulerian integral length scale $L_{w w}$ (Fig. 3e) at the measurement location. It could be argued that other scales, such as $L_{u u}$ or $L_{v v}$, would be more appropriate to normalize 
distances along $x$ and $y$, respectively. We decided however to use a same length scale for the three directions and to adopt as a reference $L_{w w}$, since this is (at any given distance from the wall) the smallest among the three (Nironi et al, 2015). First of all, we observe that for low values of $\Delta x, \Delta y$, and $\Delta z$ the local maxima of $R_{\overline{u^{\prime} c^{\prime}}}$ and $R_{\overline{w^{\prime} c^{\prime}}}$ occur at $\Delta t_{\text {lag }, o p t} \approx 0.016$ seconds and they are irrespective of the displacement direction. This value of the time delay is very similar to that estimated in previous studies performed using similar measurement systems (Contini et al, 2006; Kukačka et al, 2012; Nosek et al, 2016).

Furthermore, a large increment of $\Delta x_{i}$ produces effects depending on the space direction: vertical and transverse displacements deteriorate the correlation coefficients without affecting the optimal time lag (Figs. 8ad), whereas a longitudinal shift modifies the shape of the curves and $\Delta t_{\text {lag,opt }}$ changes significantly (Figs. $8 \mathrm{e}$ and f). In the plots we highlighted the distance where the maxima of the correlation coefficients reduce of $10 \%$ with respect to the highest value. It is worth noting that these values depend on the direction of the displacement, with $\Delta z=0.61 L_{w w}, \Delta y=0.3 L_{w w}$, and $\Delta x=0.3 L_{w w}$, for the vertical, transverse, and longitudinal shift, respectively. That means that the correlation distances depend on the space direction and the $x$ and $y$-directions are the most sensitive.

In Fig. 9 we plotted the local maxima of $R_{\overline{w^{\prime} c^{\prime}}}$ and $R \overline{u^{\prime} c^{\prime}}$ as a function of $\Delta x_{i}$ for each space direction. Results show that the $R \overline{\overline{u^{\prime} c^{\prime}}}$ is generally less sensitive than $R_{\overline{w^{\prime} c^{\prime}}}$ to variations of $\Delta x_{i}$. Concerning $R_{\overline{w^{\prime} c^{\prime}}}$, the variations in the longitudinal direction are those that affect more its decay, whereas variations along $y$ and $z$ of order $0.2 L_{w w}$ reduce only slightly its value. Based on these results we can therefore provide a general rule and fix a reference value of the distance $\Delta x_{i}$ that ensures reliable estimates of $R \overline{u_{i}^{\prime} c^{\prime}}$. Discarding the displacement in the longitudinal direction, we can assert that the distance $\Delta x_{i}$ can be set equal to $0.2 L_{w w}$, which for the present set of measurements corresponds generally to $4 \mathrm{~mm}$.

\section{Comparison with HWA-FID measurements and estimate of the mass flux balance}

We performed a cross-validation of the two systems LDA-FID and HWA-FID - by means of a comparison of the longitudinal and vertical turbulent mass fluxes computed from the experimental data. We have subsequently compared the estimates of the total longitudinal scalar flux (mean+turbulent) as measured by the two systems with the values provided by the flow meter at the source.
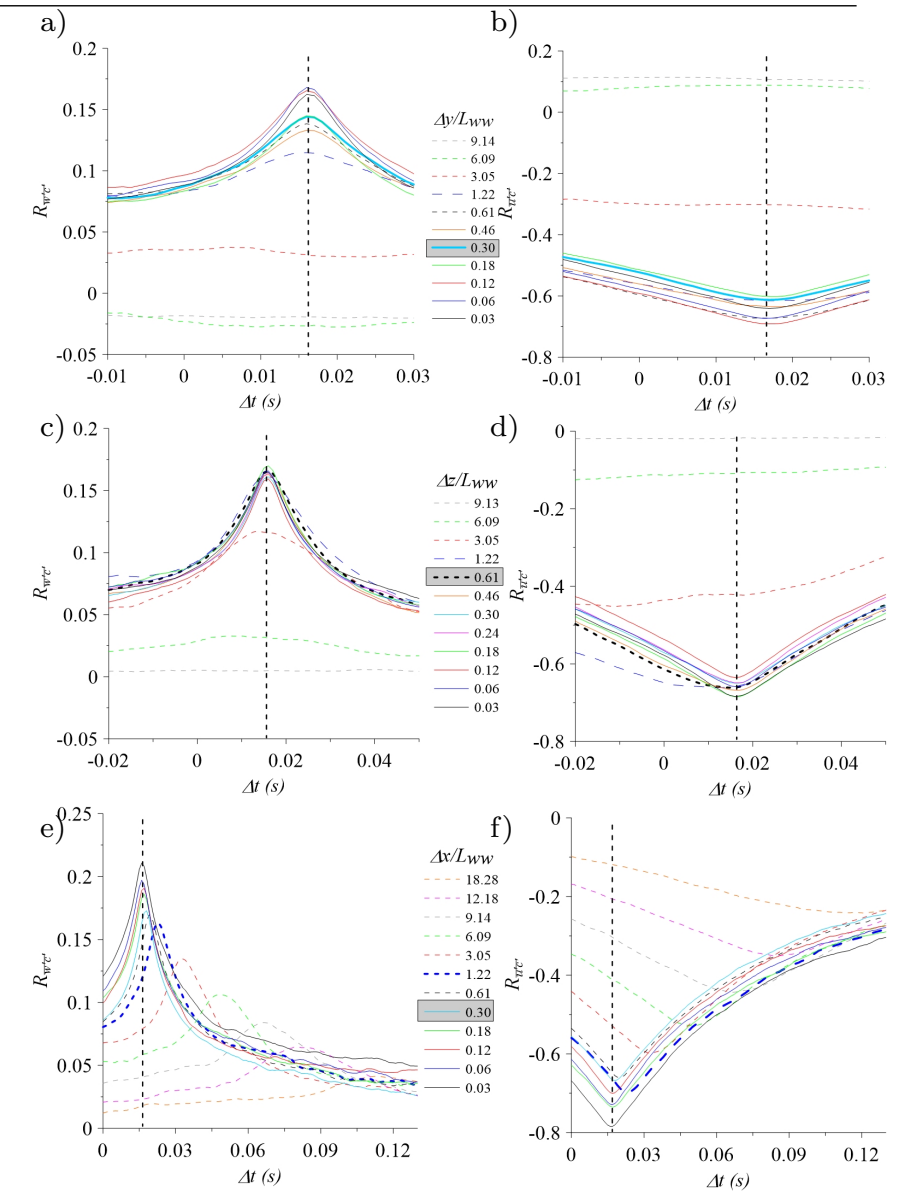

Fig. 8 Correlation coefficients vs $\Delta t_{l a g}$ for varying distance between LDA and FID: a) $R \overline{w^{\prime} c^{\prime}}$ vs $\Delta y / L_{w w}$, b) $R \overline{\bar{u}^{\prime} c^{\prime}}$ vs $\Delta y / L_{w w}$, c) $R_{\overline{w^{\prime} c^{\prime}}}$ vs $\Delta z / L_{w w}$, d) $R_{\overline{u^{\prime} c^{\prime}}}$ vs $\Delta z / L_{w w}$, e) $R \overline{\overline{w^{\prime} c^{\prime}}}$ vs $\Delta x / L_{w w}$, f) $R \overline{\overline{u^{\prime} c^{\prime}}}$ vs $\Delta x / L_{w w}$, The grey value represents the distance which induces a $10 \%$ lost on the optimal value of correlation coefficients

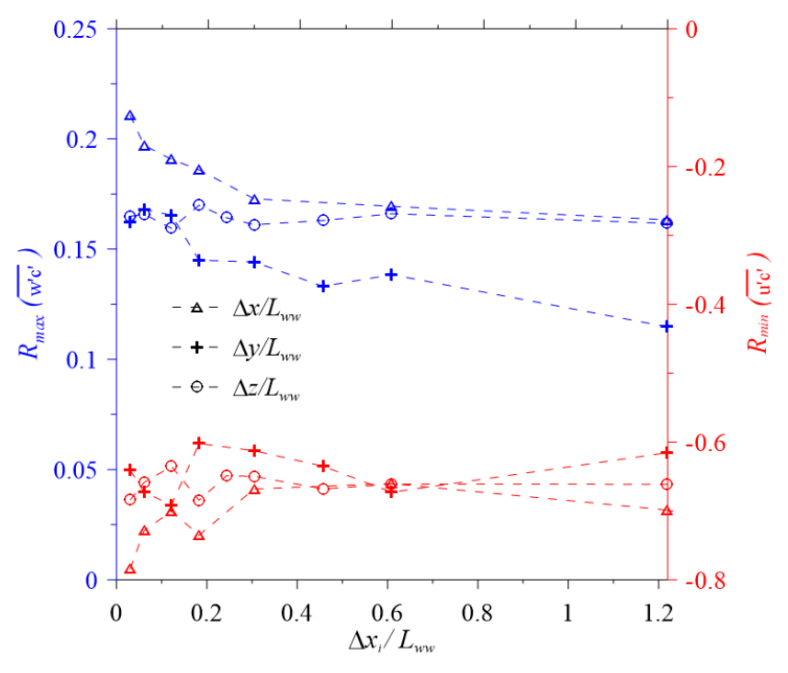

Fig. 9 Correlation coefficients vs $\Delta x_{i} / L_{w w}$ 
Note that the coupling HWA-FID does not require complex strategy of post-processing as slot correlation or $\mathrm{S}+\mathrm{H}$ technique since we can impose the same constant sampling frequency $(f=1000 \mathrm{~Hz}$ in this set of measurements) for both HWA and FID. Furthermore, the flow seeding is no more necessary and the FID measurements are not altered by the presence of oil drops. The distance between the HWA and FID was the same as that used for the LDA-FID system (about $4 \mathrm{~mm}$ ). Concerning the time lag between the two signals, we just computed the velocity-concentration correlations varying the time delay and we verified that their values were actually maximized for $\Delta t_{\text {lag }, \text { opt }}=0.016 \mathrm{~s}$.

Figure 10 shows the vertical profiles of $\overline{u^{\prime} c^{\prime}}$ and $\overline{w^{\prime} c^{\prime}}$ at increasing distances from the source. We observe that the profiles obtained with the two systems are in very good agreement one with the other. In a general way, the values provided by the LDA-FID are systematically larger than those measured by the HWA-FID. Concerning $\overline{u^{\prime} c^{\prime}}$, these discrepancies are lower than $10 \%$, and are reasonably due to the influence of seeding on the FID response that induces a slight overestimate of the concentration fluctuations (see Fig 6). Larger differences (up to $20 \%$ ) are instead observed for $\overline{w^{\prime} c^{\prime}}$. In that case, the lower values measured by the HWA-FID have also to be attributed to the tendency of the HWA in underestimating the vertical velocity fluctuations in the surface layer (see Fig. 3b) Note however that the estimates of vertical turbulent mass fluxes $\overline{w^{\prime} c^{\prime}}$ using HWA (instead of LDA) are significantly more accurate than the estimates of vertical momentum fluxes $\overline{u^{\prime} w^{\prime}}$ provided by HWA (see Fig. 3c).

An evaluation of the reliability of the measurements can be performed by computing a simple mass balance over the domain. Considering that the velocity and the scalar fields are statistically one- and two-dimensional, respectively, and that the linear source emits homogeneously:

$Q^{*}=\frac{Q_{t o t}}{L_{e f f}}=\int_{0}^{\infty} \overline{u c} d z=\int_{0}^{\infty}\left(\bar{u} \bar{c}+\overline{u^{\prime} c^{\prime}}\right) d z$,

where $Q^{*}$ is the normalized linear mass flow imposed at the release point and $L_{\text {eff }}$ is the effective width of the tunnel section, i.e. taking into account the presence of boundary layer on the lateral walls. Notably we estimated that their effect accounts for a reduction of approximately $7 \%$ of the tunnel width $L$. The longitudinal evolution of the normalized mass flow, estimated from both LDA-FID and HWA-FID measurements, is presented in Fig. 11. This shows that our estimates of $Q^{*}$ are in very good agreement with the measurements provided by the mass flow rates, with a global uncertainty of about $8 \%$ (Raupach and Legg (1983) consid- ered satisfactory differences within 15 and 20\%). The (negative) contribution of the turbulent flux $\overline{u^{\prime} c^{\prime}}$ accounts for approximately $10-15 \%$ of the total mass flux, and is affected by an uncertainty of $8-10 \%$ (almost the same for the two systems). Consequently, in absolute values, the largest uncertainty in the estimate of the total flux $Q^{*}$ is associated to the mean term $\bar{u} \bar{c}$.

Note that the accuracy of the estimates of $Q^{*}$ presented in Fig. 11 reflects also the accuracy of two features of our experimental setup, i.e. the effective homogeneity along the transversal direction $y$ of i) (the statistics of) the velocity field and ii) of the release of the scalar at the source.

\section{Conclusions}

In this study we have presented a detailed analysis on the settings of a system for the simultaneous measurements of high-frequency signals of velocity and scalar concentration in wind-tunnel experiments. This kind of measurements is essential in order to obtain direct estimates of correlation statistics, and therefore of the turbulent mass fluxes $\overline{u_{i}^{\prime} c^{\prime}}$. To these purposes we focused on data acquired by the coupling of two different anemometers, LDA and HWA, with a FID (for concentration measurements). Wind-tunnel experiments simulated the dispersion downwind a ground-level source within a neutral turbulent boundary layer over a rough surface. By means of a parametric study we identified the optimal settings of a LDA-FID system, notably for what concerns the distance between the measurement volumes of the two instruments allowing reliable measurements to be performed without the velocity field is perturbed by the FID. Furthermore, we identified the time lag with which the signals are collected and we tested two different techniques - slot correlation and sample-and-hold reconstruction and resampling (applied to the concentration signals) - to evaluate the cross-correlation between the velocity and scalar signals. We showed that the two methods provide very similar estimates of the turbulent mass fluxes with differences lower than $6 \%$.

We also focused on the effects of mobilised olive oil droplets (used here to seed the flow for the LDA measurements) on the repeatability and accuracy of concentration sampling performed by the FID. The presence of seeding particles induces a slight larger dispersion of the concentration values and an increase of the uncertainty in the measurements that, however, does not exceed $5 \%$. We compared the measurements of the turbulent scalar fluxes $\overline{u^{\prime} c^{\prime}}$ and $\overline{w^{\prime} c^{\prime}}$ obtained by the two systems, i.e. LDA-FID and HWA-FID, and discussed the existing differences in spite of a general good agreement. Fi- 


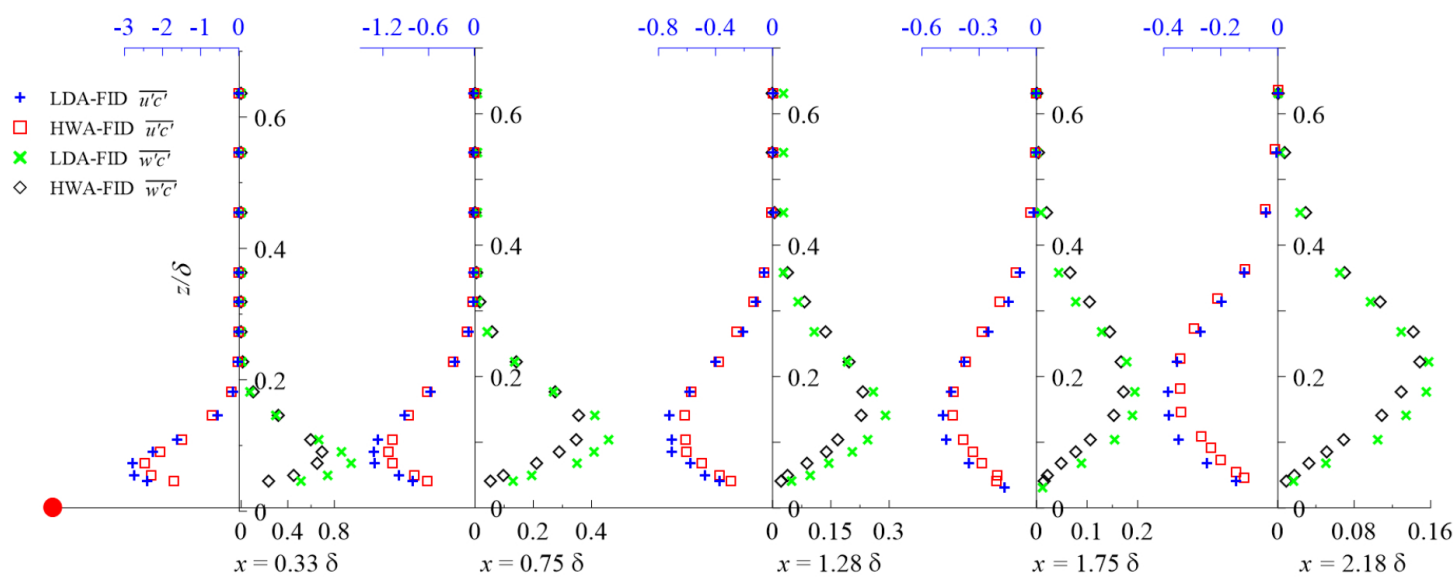

Fig. 10 Vertical profiles of longitudinal and vertical turbulent mass fluxes varying the distance from the source location

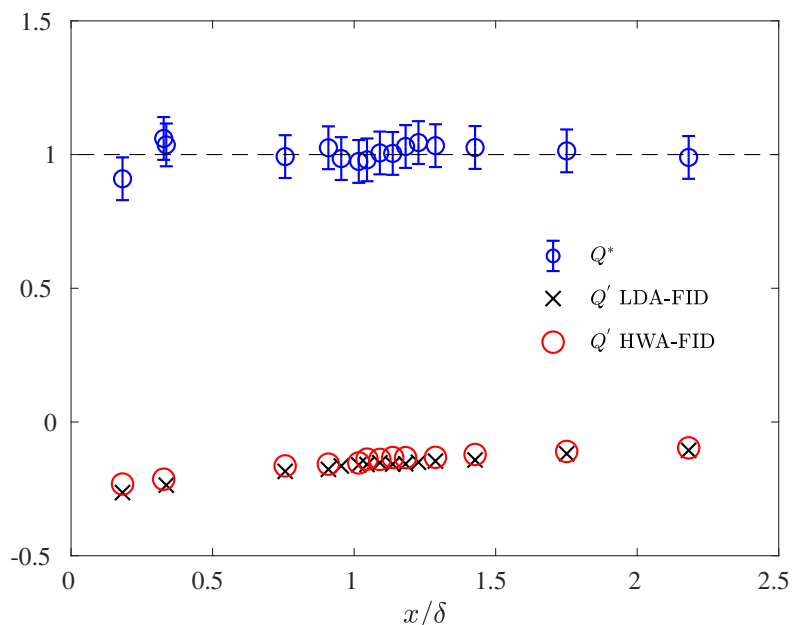

Fig. 11 Longitudinal evolution of the normalized total mass flux $Q^{*}$ (Eq. 7), and turbulent mass fluxes measured by means of HWA-FID and LDA-FID

nally, we estimated the longitudinal mass flux obtained by integrating the vertical profiles of $\overline{u c}$ with that provided by a flow meter at the source (of ethane). The good agreement between the two values of the scalar flux demonstrates further the reliability of such measurement system.

\section{References}

Adrian R (1983) Laser velocimetry. In: Goldstein R (ed) Fluid mechanics measurements, Hemisphere, Washington D.C., chap 5, pp 175-301

Adrian RJ, Yao CS (1987) Power spectra of fluid velocities measured by laser Doppler velocimetry. Exp Fluids 5(1):17-28
Boskou D, Blekas G, Tsimidou M (2006) 4 - Olive oil composition. In: Boskou D (ed) Olive Oil, second edition edn, AOCS Press, pp $41-72$

Carpentieri M, Robins AG (2010) Tracer flux balance at an urban canyon intersection. Boundary Layer Meteorol 135(2):229-242

Carpentieri M, Hayden P, Robins AG (2012) Wind tunnel measurements of pollutant turbulent fluxes in urban intersections. Atmos Environ 46:669-674

Contini D, Hayden P, Robins AG (2006) Concentration field and turbulent fluxes during the mixing of two buoyant plumes. Atmos Environ 40:7842-7857

Edwards RV (1987) Report of the Special Panel on Statistical Particle Bias Problems in Laser Anemometry. J Fluids Eng 109(2):89-93

Fackrell JE (1980) A flame ionisation detector for measuring fluctuating concentration. J Phys E: Sci Instrum 13(8):888-893

Fackrell JE, Robins AG (1982) Concentration fluctuations and fluxes in plumes from point sources in a turbulent boundary layer. J Fluid Mech 117:1-26

George WK (1988) Quantitative measurement with the burst-mode laser doppler anemometer. Exp Therm Fluid Sci 1(1):29 - 40

Iacobello G, Marro M, Ridolfi L, Salizzoni P, Scarsoglio S (2019) Experimental investigation of vertical turbulent transport of a passive scalar in a boundary layer: Statistics and visibility graph analysis. Phys Rev Fluids 4:104,501

Irwin HPAH (1981) The design of spires for wind simulation. J Wind Eng Ind Aerod 7:361-366

Jensen KD (2004) Flow measurements. Journal of the Brazilian Society of Mechanical Sciences and Engineering 26:400 - 419 
Jimènez J (2004) Turbulent flows over rough wall. Annu Rev Fluid Mech 36:173-96

Joint Committee for Guides in Metrology (2008) Jcgm 100: Evaluation of measurement data - guide to the expression of uncertainty in measurement. Tech. rep., JCGM

Jorgensen FE (2002) How to measure turbulence with hot-wire anemometers - a practical guide. Tech. rep., Dantec Dynamics

Kawall JG, Shokr M, Keffer JF (1983) A digital technique for the simultaneous measurement of streamwise and lateral velocities in turbulent flows. J Fluid Mech 133:83-112

Koeltzsch K (1999) The height dependence of the turbulent Schmidt number within the boundary layer. Atmos Environ 34(7):1147-1151

Kukačka L, Nosek S, Kellnerová R, Jurčáková K, Jaňour Z (2012) Wind tunnel measurement of turbulent and advective scalar fluxes: A case study on intersection ventilation. Sci World J Article ID 381357

Liao Q, Cowen EA (2002) The information content of a scalar plume - a plume tracing perspective. Environ Fluid Mech 2(1):9-34

Marucci D, Carpentieri M (2020) Dispersion in an array of buildings in stable and convective atmospheric conditions. Atmos Environ 222:117,100

Mayo WTJ (1975) Modeling laser velocimeter signals as triply stochastic Poisson processes. In: Laser Anemometry, pp 455-484

Metzger MM, Klewicki JC (2003) Development and characterization of a probe to measure scalar transport. Meas Sci Technol 14(8):1437-1448

Müller E, Nobach H, Tropea C (1998) A refined reconstruction-based correlation estimator for twochannel, non-coincidence laser Doppler anemometry. Meas Sci Technol 9(3):442-451

Nironi C, Salizzoni P, Marro M, Mejan P, Grosjean N, Soulhac L (2015) Dispersion of a passive scalar from a point source in a turbulent boundary layer. Part I: Velocity and cocnetration measurements. BoundaryLayer Meteorol 156:415-446

Nobach H (2016) Present methods to estimate the cross-correlation and cross-spectral density for twochannel laser Doppler anemometry. In: 18th International Symposium on the Application of Laser and Imaging Techniques to Fluid Mechanics, Lisbon, Portugal, 4-7 July

Nobach H, Müller E, Tropea C (1998) Efficient estimation of power spectral density from laser Doppler anemometer data. Exp Fluids 24(5):499-509

Nosek S, Kukačka L, Kellnerová R, Jurčáková K, Jaňour Z (2016) Ventilation processes in a threedimensional street canyon. Boundary-Layer Meteorol
159(2):259-284

Perry AE, Lim KL, Henbest SM (1987) An experimental study of the turbulence structure in smooth- and rough-wall boundary layers. J Fluid Mech 177:437466

Pope S (2013) Turbulent flows. Cambridge university press

Raupach MR, Legg BJ (1983) Turbulent dispersion from an elevated line source: measurements of windconcentration moments and budgets. J Fluid Mech 136:111-137

Robins AG (1979) The development and structure of simulated neutrally stable atmospheric boundary layers. J Ind Aerodyn 4:71-100

Schlichting H (1979) Boundary layer theory. Springer, Berlin

Stapountzis H, Sawford BL, Hunt JCR, Britter RE (1986) Structure of the temperature field downwind of a line source in grid turbulence. J Fluid Mech 165:401-424

Talluru KM, Philip J, Chauhan KA (2018) Local transport of passive scalar released from a point source in a turbulent boundary layer. J Fluid Mech 846:292-317

Tennekes H (1982) Similarity relations, scaling laws and spectral dynamics. Atmospheric Turbulence and Air Pollution Modelling pp 37-68

Tutu NK, Chevray R (1975) Cross-wire anemometry in high intensity turbulence. J Fluid Mech 71:785-800

Velte CM, George WK, Buchhave P (2014) Estimation of burst-mode LDA power spectra. Exp Fluids $55(3): 1674$

Vinçont J, Simoëns S, Ayrault M, Wallace J (2000) Passive scalar dispersion in a turbulent boundary layer from a line source at the wall and downstream of an obstacle. J Fluid Mech 424:127

Yaacob M, Schlander R, Buchhave P, Velte C (2020) A novel laser doppler anemometer (lda) for highaccuracy turbulence measurements. Exp Fluids 